\title{
Allergikerinnen werden schneller schwanger
}

\author{
Beim Eintritt einer Schwangerschaft kommt es - wie auch bei der \\ Entstehung einer Allergie - zu einer Verschiebung der Immun- \\ antwort vom Typ TH1 zum Typ TH2. Dies soll die Einnistung des \\ Embryos in der Gebärmutter erleichtern. Könnte es also sein, dass \\ allergische Frauen leichter in ,andere Umstände“ kommen?
}

\begin{abstract}
$\mathrm{D}$ änische Epidemiologen waren einem Zusammenhang zwischen allergischer Rhinitis bei Frauen und der Wahrscheinlichkeit einer Schwangerschaft auf der Spur. Bereits 1997 hatte eine Studie ergeben, dass Mütter mit einer atopischen Erkrankung im Mittel mehr Kinder haben als Mütter ohne ein solches Leiden in der Anamnese. Vermutet wurde, dass es bei gesunden Frauen durch die normale TH1-vermittelte Immunantwort öfter zur Abstoßung eines Embryos kommt als bei allergischen Frauen, bei denen diese Art der Immunreaktion unterdrückt ist.
\end{abstract}

Nun wurden die Daten von über 31.000 Teilnehmerinnen einer großen dänischen Geburtskohorten-Studie ausgewertet. Insgesamt litten $13,5 \%$ der Mütter an einer allergischen Rhinitis, darunter mehr Erstgebärende als Frauen, die bereits zuvor einmal schwanger gewesen waren. Entgegen der Erwartung unterschieden sich Allergikerinnen und gesunde Frauen nicht in der Häufigkeit spontaner Aborte. Bei den Frauen, die ihre Schwangerschaft bewusst geplant hatten, waren es allerdings diejenigen mit allergischer Rhinitis, die häufiger bereits innerhalb eines Jahres
Mutterfreuden entgegen sehen konnten (Odds Ratio 1,18; 95\%-Konfidenzintervall 1,06-1,32).

Die Autoren sehen ihre Hypothese damit nur teilweise bestätigt: Das bereits auf $\mathrm{TH} 2$-Reaktionen umgestellte Immunsystem - beziehungsweise seine Bereitschaft zur rascheren Umstellung - scheint bei Allergikerinnen zwar insgesamt den Beginn einer Schwangerschaft $\mathrm{zu}$ erleichtern, wirkt sich aber nicht merklich begünstigend auf deren weiteren Verlauf und erfolgreichen Ausgang aus.

Fazit: Frauen mit allergischer Rhinitis werden im Vergleich zu Nicht-Allergikerinnen leichter schwanger, ihre Chancen, das Kind auszutragen, sind jedoch nicht signifikant größer. Ursache könnten die typischen immunologischen Veränderungen in Folge einer Allergie sein

isi

Westergaard T et al. Reproductive history and allergic rhinitis among 31145 Danish women. Clin Exp Allergy 2003; 33: 301-5

\section{Schützt Stillen vor Asthma?}

\section{Stillen kann Kinder vor Bronchitis und pfeifenden Atemgeräuschen schützen. Dies zeigen verschiedene Studien. Die Ergebnisse zu Asthma sind jedoch bisher uneinheitlich. Anhand von Daten aus einer repräsentativen, epidemiologischen Studie gingen Forscher der amerikanischen National Institutes of Health dieser Frage nun erneut auf den Grund.}

$\mathrm{P}$ atricia Chulada und ihr Team werteten die Daten von 7.766 Kindern im Alter zwischen 2 Monaten und 6 Jahren aus, von denen bekannt war, ob und wie lange sie von ihren Müttern gestillt worden waren. Diese Angaben setzten sie in Beziehung zu Auskünften der Eltern, wie häufig diese bei ihren Kindern im Laufe der vorausgegangen 12 Monate pfeifenden Atem bemerkt hatten beziehungsweise ob von einem Arzt eine Asthmadiagnose gestellt worden war. Weiter wurde dokumentiert, ob die Eltern unter Asthma oder Heuschnupfen litten, ob im häuslichen Umfeld des Kindes Raucher lebten und ob die Mutter während der Schwangerschaft geraucht hatte.

Eine multivariate Analyse unter Einbeziehung von Korrekturfaktoren ergab ein differenzierteres Bild als erwartet: Muttermilch verringert nicht allgemein, sondern nur bis zu einem Alter von 24 Monaten signifikant das Risiko, an Asthma oder verengten Bronchien zu erkranken. Als mögliche Erklärung hierfür diskutieren die Autoren, dass das Stillen nur vorübergehend

Statistisch hat das Kind auf dem ganz rechten Foto zumindest in der ersten beiden Lebensjahren ein reduziertes Risiko, an Asthma zu erkranken. vor einer allergischen Sensibilisierung schützt, weil der Kontakt mit potenziellen Nahrungsallergenen zeitlich hinausgeschoben wird und die Muttermilch Substanzen enthält, die die Entwicklung und Reifung des Immunsystems fördern.

Überrascht zeigten sie sich über den Befund, dass offenbar besonders die Kinder von den Vorteilen des Stil-

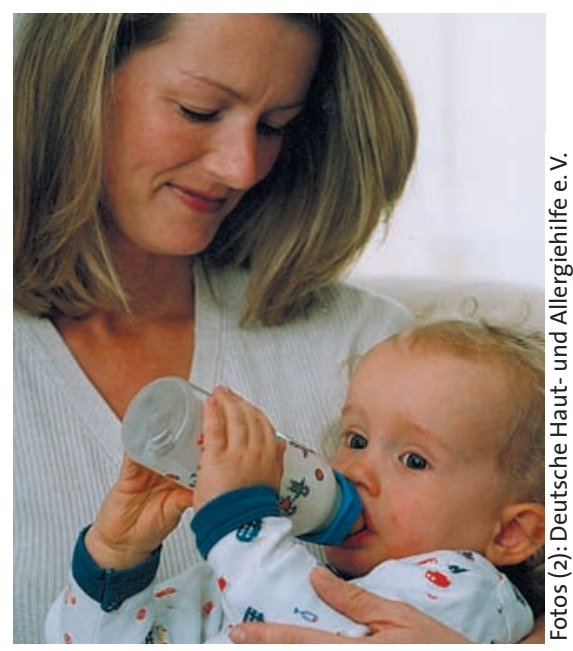

